FACTA UNIVERSITATIS (NIŠ)

Ser. Math. Inform. Vol. 35, No 5 (2020), 1315-1325

https://doi.org/10.22190/FUMI2005315A

\title{
SURFACE FAMILY WITH COMMON LINE OF CURVATURE IN 3-DIMENSIONAL GALILEAN SPACE
}

\author{
Mustafa Altin and İnan Ünal
}

(C) 2020 by University of Niš, Serbia | Creative Commons Licence: CC BY-NC-ND

\begin{abstract}
In this paper we tried to find parametric presentation of a surface family with common line of curvature in 3-dimensional Galilean space. We have obtained necessary and sufficient conditions for the curve to be a common line of curvature on this surface. We have stated examples to visualize our results and also, we have examined a torsion free curve.
\end{abstract}

Keywords: surface family; curvature; 3-dimensional Galilean space.

\section{Introduction}

The surface family (or pencil surface) is a notion in differential geometry applied in engineering science such as computer, manufacturing, mechanical engineering [25]. In 2004 Wang et al. [25] gave the definition of a surface family. Their paper is a reverse engineering problem to find a spatial curve to characterize the surface and also the paper contains conditions for a curve to be a geodesic on this surface. Besides, their work could be seen as an example of industrial mathematics. Kasap et al. [10] generalized this study by assumption of more general marchingscale functions. In [13] $\mathrm{Li}$ et al studied the approximation minimal surface with geodesics by using Dirichlet function and they minimized the area of surface family by using Dirichlet approach. This method can be used for obtaining minimal cost of material while building surfaces. The surface family notion has been studied by many researchers $[1,2,9,10]$.

There are many special curves on a surface such as geodesics. One of them is the line of curvature. A line of curvature is a curve on a surface whose tangent line at every point is aligned along a principal curvature direction. In [4] Che at al. analysed and computed these curves which are defined on implicit surface and worked on differential geometry of them. Same authors derived a necessary and

Received September 02, 2020; accepted October 10, 2020

2020 Mathematics Subject Classification. Primary 53A35; Secondary 53B32,53C42 
sufficient condition for a given curve to be the line of curvature on the surface. Surface family with common line of curvature has been studied in $[7,8,12]$.

Galileo geometry is a type of non-Euclidean geometry based on Galileo principle of relativity [20] and it has many important applications in physics [14]. In the last decades, these kind of spaces have become interesting by geometers because of their significant properties as a non-Euclidean geometry. Curves and surfaces in Galilean geometry has been studied by many authors $[3,5,6,15-17,21]$. Surfaces family, especially, in Galilean space have been studied in [22-24].

In this study, we examined a surface family with common line of curvature in 3 - dimensional Galilean space. We obtain necessary and sufficient conditions for the curve to be a line of curvature on the surface. We get some results for a torsion free curve. Finally, we present examples and plot their graphs.

\section{Preliminaries}

A. Cayley and F. Klein discovered that both Euclidean and non-Euclidean geometries can be considered as mathematical structures living inside projectivemetric spaces. Their contribution to geometry is called Cayley-Klein geometry and non- Euclidean geometries could be classified by this geometry. In fact, the 3 -dimensional Galilean geometry is also a Cayley-Klein space [20].

\subsection{Basic Facts in 3D Galilean Space}

In this subsection, we recall some fundamental facts from Galilean geometry. For details see $[18,20]$.

A vector $\omega=\left(\omega_{1}, \omega_{2}, \omega_{3}\right)$ in 3 -dimensional Galilean space $\mathbb{G}_{3}$ is called nonisotropic if $\omega_{1} \neq 0$, otherwise it is called isotropic.

Let $\omega=\left(\omega_{1}, \omega_{2}, \omega_{3}\right)$ and $\eta=\left(\eta_{1}, \eta_{2}, \eta_{3}\right)$ be two vectors in Galilean space $\mathbb{G}_{3}$. The inner product and the vector product of $\omega$ and $\eta$ in $\mathbb{G}_{3}$ are defined by

$$
\langle\omega, \eta\rangle=\left\{\begin{array}{c}
\omega_{1} \eta_{1}, \text { if } \omega_{1} \neq 0 \text { or } \eta_{1} \neq 0 \\
\omega_{2} \eta_{2}+\omega_{3} \eta_{3} \text { if } \omega_{1}=0 \text { and } \eta_{1}=0
\end{array}\right.
$$

and

$$
\omega \times \eta= \begin{cases}\left|\begin{array}{ccc}
e_{1} & e_{2} & e_{3} \\
0 & \omega_{2} & \omega_{3} \\
0 & \eta_{2} & \eta_{3}
\end{array}\right| & \text { if } \omega_{1}=\eta_{1}=0, \\
\left|\begin{array}{ccc}
0 & e_{2} & e_{3} \\
\omega_{1} & \omega_{2} & \omega_{3} \\
\eta_{1} & \eta_{2} & \eta_{3}
\end{array}\right| & \text { if } \omega_{1} \neq 0 \text { or } \eta_{1} \neq 0 .\end{cases}
$$

respectively. 
Let $\gamma: I \rightarrow \mathbb{G}_{3}, \quad I \subset \mathbb{R}$ be a curve in $\mathbb{G}_{3}$ given by $\gamma(\phi)=(\delta(\phi), \zeta(\phi), \psi(\phi))$. Then the curvature $\kappa_{1}$ and torsion $\kappa_{2}$ of $\gamma(\phi)$ is obtained as

$$
(2.1) \kappa_{1}(\phi)=\left\|\gamma^{\prime \prime}(\phi)\right\|, \quad \kappa_{2}(\phi)=\frac{1}{\kappa_{1}^{2}(\phi)} \operatorname{det}\left(\gamma^{\prime}(\phi), \gamma^{\prime \prime}(\phi), \gamma^{\prime \prime \prime}(\phi)\right), \kappa_{1}(\phi) \neq 0
$$

where $\|$,$\| is the Galilean norm. Thus, we have Frenet formulas of \gamma(\phi)$ by

$$
\left\{\begin{array}{c}
V_{1}{ }^{\prime}=\kappa_{1} V_{2} \\
V_{2}{ }^{\prime}=\kappa_{2} V_{3} \\
V_{3}{ }^{\prime}=-\kappa_{2} V_{2}
\end{array}\right.
$$

where $V_{1}, V_{2}$ and $V_{3}$ are tangent, normal and binormal vector fields of $\gamma(\phi)$, respectively.

If $\delta^{\prime}(\phi)=0$, then $\gamma(\phi)$ is called a non-admissible curve, otherwise it is called an admissible curve. Let $\gamma(\phi)$ be an admissible curve in $\mathbb{G}_{3}$, given by

$$
\gamma(\phi)=(\phi, \zeta(\phi), \psi(\phi)) .
$$

Then $\kappa_{1}$ and $\kappa_{2}$ can be obtained as

$$
\kappa_{1}(\phi)=\sqrt{\zeta^{\prime \prime}(\phi)^{2}+\psi^{\prime \prime}(\phi)^{2}}, \kappa_{2}(\phi)=\frac{1}{\left(\kappa_{1}(\phi)\right)^{2}} \operatorname{det}\left(\gamma^{\prime}(\phi), \gamma^{\prime \prime}(\phi), \gamma^{\prime \prime \prime}(\phi)\right)
$$

and the Frenet vectors are given by

$$
\left\{\begin{array}{c}
V_{1}(\phi)=\gamma^{\prime}(\phi)=\left(1, \zeta^{\prime}(\phi), \psi^{\prime}(\phi)\right), \\
V_{2}(\phi)=\frac{\gamma^{\prime \prime}(\phi)}{\kappa_{1}(\phi)}=\frac{1}{\kappa_{1}(\phi)}\left(0, \zeta^{\prime \prime}(\phi), \psi^{\prime \prime}(\phi)\right), \\
V_{3}(\phi)=\frac{1(\phi)}{\kappa_{1}(\phi)}\left(0,-\psi^{\prime \prime}(\phi), \zeta^{\prime \prime}(\phi)\right) .
\end{array}\right.
$$

\subsection{Some facts on Surface Theory in 3D Galilean Space}

A surface in $\mathbb{G}_{3}$ is a parametric mapping from a region $R$ in $\mathbb{R}^{2}$ to $\mathbb{G}_{3}$ such as

$$
S: R \subset \mathbb{R}^{2} \rightarrow \mathbb{G}_{3}, \quad S(\phi, \varphi)=\left(S_{1}(\phi, \varphi), S_{2}(\phi, \varphi), S_{3}(\phi, \varphi)\right)
$$

where $S_{1}, S_{2}$ and $S_{3}$ are functions in $C^{1}\left(\mathbb{G}_{3}, \mathbb{R}\right)$. The normal vector field of $S$ is given by

$$
\mathcal{N}(\phi, \varphi)=S_{\phi} \times S_{\varphi}
$$

where $S_{\phi}=\frac{\partial S}{\partial \phi}$ and $S_{\varphi}=\frac{\partial S}{\partial \varphi}$ are partial derivatives of $S$.

Every surface has its own intrinsic geometry which has been known since Gauss. So, curves on a surface have geometric properties independent from the ambient space. We have a classification for curves on a surface by following definition.

Definition 2.1. Let $\gamma(\phi)$ be a curve on a surface $S$ in 3-dimensional Galilean space $\mathbb{G}_{3}$. Then $\gamma(\phi)$ is 
1. a line of curvature, if the tangent vector at any point is in the direction of the principal curvature.

2. a geodesic if the normal vector field $V_{2}(\phi)$ of the curve $\gamma(\phi)$ and the normal $\mathcal{N}\left(\phi, \varphi_{0}\right)$ are parallel.

3. an asymptotic if the the binormal $V_{3}(\phi)$ of $\gamma(\phi)$ and the normal $\mathcal{N}\left(\phi, \varphi_{0}\right)$ of the surface at any point on $\gamma(\phi)$, are parallel to each other.

On the other hand, if $\gamma(\phi)$ is both an asymptotic and a parametric (isoparametric) curve, then it is called isoasymptotic; if it is both an geodesic and a parametric (isoparametric) curve, then it is called isogeodesic.

The well-known theorem below gives the conditions for any curve on a surface $S$ to be the line of curvature. For proof and details, we refer to reader [19].

Theorem 2.1. (Monge's Theorem) A necessary and sufficient condition for a curve on a surface to be a line of curvature is that the surface normals along the curve form a developable surface [19].

Let $S(\phi, \varphi)$ be a parametric surface in $\mathbb{G}_{3}$ is defined as follow;

$$
S(\phi, \varphi)=\gamma(\phi)+\left[\lambda_{1}(\phi, \varphi) V_{1}(\phi)+\lambda_{2}(\phi, \varphi) V_{2}(\phi)+\lambda_{3}(\phi, \varphi) V_{3}(\phi)\right]
$$

for $(\phi, \varphi) \in R=\left[I_{1}, I_{2}\right] \times\left[I_{3}, I_{4}\right]$, where $\lambda_{1}(\phi, \varphi), \lambda_{2}(\phi, \varphi)$ and $\lambda_{3}(\phi, \varphi)$ are the values of the marching-scale functions in $C^{1}(S, \mathbb{R})$ and $\left\{V_{1}(\phi), V_{2}(\phi), V_{3}(\phi)\right\}$ is the Frenet frame of $\gamma(\phi)$. The surface (2.6) is called surface family with a common curve $\gamma(\phi)$.

A ruled surface formed by the surface normals can be given by

$$
\Psi(\phi, \varphi)=\gamma(\phi)+\varphi \mathfrak{n},
$$

where $\varphi$ is the distance of a point on $\Psi(\phi, \varphi)$ to point $\gamma(\phi)$ and $\mathfrak{n}=\cos \theta V_{2}(\phi)+$ $\sin \theta V_{3}(\phi)$, the vector functions $V_{2}(\phi), V_{3}(\phi)$ are the principal normal and the binormal of $\gamma(\phi)$, respectively. The surface $\Psi(\phi, \varphi)$ is called a normal surface [11].

Thus, by Monge's Theorem, $\gamma(\phi)$ is the line of curvature if and only if $\Psi(\phi, \varphi)$ is developable and $\mathfrak{n}$ is parallel to the normal vector field $\mathcal{N}$ of the surface (2.6). Also by classical differential geometry, it is well known that a surface is developable if and only if $\operatorname{det}\left(\gamma^{\prime}(\phi), \mathfrak{n}, \mathfrak{n}^{\prime}\right)=0$ (see [19]).

Hence from (2.2), we get

$$
\left|\begin{array}{ccc}
1 & 0 & 0 \\
0 & \cos \theta & \sin \theta \\
0 & -\theta^{\prime} \sin \theta-\kappa_{2} \sin \theta & \kappa_{2} \cos \theta+\theta^{\prime} \cos \theta
\end{array}\right|=0
$$

and so

$$
\theta^{\prime}+\kappa_{2}=0
$$


This means that

$$
\theta=-\int_{\phi_{0}}^{\phi} \kappa_{2} d \phi+\theta_{0}
$$

where $\phi_{0}$ is the starting value of arc length and $\theta_{0}=\theta_{0}\left(\phi_{0}\right)$. In this paper, we assume $\phi_{0}=0$. Then by substituting $\theta$ in $\mathfrak{n}$ and with parallelity of $\mathfrak{n}$ to $\mathcal{N}$, we obtain the result that $\gamma(\phi)$ is the line of curvature.

\section{Surfaces with common line of curvature in 3D Galilean space $\mathbb{G}_{3}$}

In this section, we work on surfaces family in Galilean 3-space $\mathbb{G}_{3}$. We give if and only if conditions for a unit speed non-isotropic curve, being a line of curvature on a surface family. Furthermore, we give some examples and we present their graphics.

Theorem 3.1. The curve $\gamma(\phi)=(\phi, \zeta(\phi), \psi(\phi))$ is a line of curvature on the surface defined in (2.6) if and only if

$$
\left\{\begin{array}{c}
\lambda_{1}\left(\phi, \varphi_{0}\right)=\lambda_{2}\left(\phi, \varphi_{0}\right)=\lambda_{3}\left(\phi, \varphi_{0}\right)=0 \\
-\frac{\partial \lambda_{3}\left(\phi, \varphi_{0}\right)}{\partial \varphi}=\mu(\phi) \cos \theta, \frac{\partial \lambda_{2}\left(\phi, \varphi_{0}\right)}{\partial \varphi}=\mu(\phi) \sin \theta
\end{array}\right.
$$

where $(\phi, \varphi) \in R=\left[I_{1}, I_{2}\right] \times\left[I_{3}, I_{4}\right], \quad \mu(\phi) \neq 0$. The functions $\theta(\phi)$ and $\mu(\phi)$ are called controlling functions.

Proof. Let $S(\phi, \varphi)$ be a surface in $\mathbb{G}_{3}$ given by (2.6). For a curve $\gamma(\phi)$ on $S(\phi, \varphi)$ which is isoparametric, we have a parameter $\varphi_{0} \in\left[I_{3}, I_{4}\right]$ such that $\gamma(\phi)=S\left(\phi, \varphi_{0}\right)$ with conditions

$$
\lambda_{1}\left(\phi, \varphi_{0}\right)=\lambda_{2}\left(\phi, \varphi_{0}\right)=\lambda_{3}\left(\phi, \varphi_{0}\right)=0,\left(\phi, \varphi_{0}\right) \in R .
$$

By direct computations, we have

$$
\begin{aligned}
\frac{\partial S(\phi, \varphi)}{\partial \phi} & =\left[1+\frac{\partial \lambda_{1}(\phi, \varphi)}{\partial \phi}\right] V_{1}(\phi) \\
& +\left[\kappa_{1} \lambda_{1}(\phi, \varphi)+\frac{\partial \lambda_{2}(\phi, \varphi)}{\partial \phi}-\kappa_{2} \lambda_{3}(\phi, \varphi)\right] V_{2}(\phi) \\
& +\left[\kappa_{2} \lambda_{2}(\phi, \varphi)+\frac{\partial \lambda_{3}(\phi, \varphi)}{\partial \phi}\right] V_{3}(\phi)
\end{aligned}
$$

and

$$
\frac{\partial S(\phi, \varphi)}{\partial \varphi}=\frac{\partial \lambda_{1}(\phi, \varphi)}{\partial \varphi} V_{1}(\phi)+\frac{\partial \lambda_{2}(\phi, \varphi)}{\partial \varphi} V_{2}(\phi)+\frac{\partial \lambda_{3}(\phi, \varphi)}{\partial \varphi} V_{3}(\phi)
$$


Thus, we get the normal vector of surface by

$$
\mathcal{N}(\phi, \varphi)=\frac{\partial S(\phi, \varphi)}{\partial \phi} \times \frac{\partial S(\phi, \varphi)}{\partial \varphi}
$$

So, for $\varphi_{0} \in\left[I_{3}, I_{4}\right]$, we have

$$
\mathcal{N}\left(\phi, \varphi_{0}\right)=\mathcal{N}_{1}\left(\phi, \varphi_{0}\right) V_{1}(\phi)+\mathcal{N}_{2}\left(\phi, \varphi_{0}\right) V_{2}(\phi)+\mathcal{N}_{3}\left(\phi, \varphi_{0}\right) V_{3}(\phi)
$$

where

$$
\begin{aligned}
\mathcal{N}_{1}\left(\phi, \varphi_{0}\right) & =0 \\
\mathcal{N}_{2}\left(\phi, \varphi_{0}\right) & =\frac{\partial \lambda_{1}\left(\phi, \varphi_{0}\right)}{\partial \varphi}\left(\kappa_{2} \lambda_{2}\left(\phi, \varphi_{0}\right)+\frac{\partial \lambda_{3}\left(\phi, \varphi_{0}\right)}{\partial \phi}\right)-\left(1+\frac{\partial \lambda_{1}\left(\phi, \varphi_{0}\right)}{\partial \phi}\right) \frac{\partial \lambda_{3}\left(\phi, \varphi_{0}\right)}{\partial \varphi} \\
\mathcal{N}_{3}\left(\phi, \varphi_{0}\right) & =\left(1+\frac{\partial \lambda_{1}\left(\phi, \varphi_{0}\right)}{\partial \phi}\right) \frac{\partial \lambda_{2}\left(\phi, \varphi_{0}\right)}{\partial \varphi}-\left(\kappa_{1} \lambda_{1}\left(\phi, \varphi_{0}\right)\right. \\
& \left.+\frac{\partial \lambda_{2}\left(\phi, \varphi_{0}\right)}{\partial \phi}-\kappa_{2} \lambda_{3}\left(\phi, \varphi_{0}\right)\right) \frac{\partial \lambda_{1}\left(\phi, \varphi_{0}\right)}{\partial \varphi} .
\end{aligned}
$$

Suppose that $\gamma(\phi)$ is a line of curvature on $S(\phi, \varphi)$. Thus, for a function $\mu(\phi) \neq 0$ on $S(\phi, \varphi)$, necessary and sufficient condition to provide $\mathfrak{n}(\phi) \| \mathcal{N}\left(\phi, \varphi_{0}\right)$ is

$$
\begin{array}{ll}
\mathcal{N}_{2}\left(\phi, \varphi_{0}\right) & =\mu(\phi) \cos \theta \\
\mathcal{N}_{3}\left(\phi, \varphi_{0}\right) & =\mu(\phi) \sin \theta .
\end{array}
$$

So the proof is completed.

Example 3.1. Let $\gamma(\phi)=(\phi, 2 \sin (\phi), 2 \cos (\phi))$ be an admissible curve in $\mathbb{G}_{3}$. Then, we get the first and the second curvatures of $\gamma(\phi)$ by $\kappa_{1}=2$ and $\kappa_{2}=-1$. Thus, the Frenet frame is obtained by

$V_{1}(\phi)=(1,2 \cos (\phi),-2 \sin (\phi)), V_{2}(\phi)=(0,-\sin (\phi),-\cos (\phi)), V_{3}(\phi)=(0, \cos (\phi),-\sin (\phi))$.

If we choose

$$
\lambda_{1}(\phi, \varphi)=\phi^{2} \varphi, \lambda_{2}(\phi, \varphi)=\phi \sin (\theta) \sin (\phi \varphi), \lambda_{3}(\phi, \varphi)=\cos (\theta)\left(\phi-\phi e^{\phi \varphi}\right),
$$

then we get surface family $S(\phi, \varphi)$ given by (2.6) with common curve $\gamma(\phi)$. Then, by taking $\mu(\phi)=\phi^{2}$ and $\varphi_{0}=0$, the conditions given in (3.1) are satisfied.

Suppose that the normal surface $\Psi(\phi, \varphi)$ of $S$ is developable in $\mathbb{G}_{3}$. From (2.8), we have $\theta=\phi$. Thus, we get

$$
\begin{array}{ll}
S_{1}(\phi, \varphi) & =\phi+\phi^{2} \varphi \\
S_{2}(\phi, \varphi) & =2 \sin (\phi)+2 \phi^{2} \varphi \cos (\phi)+\sin ^{2}(\phi) \phi \sin (\phi \varphi)+\cos ^{2}(\phi)\left(\phi-\phi e^{\phi \varphi}\right) \\
S_{3}(\phi, \varphi) & =2 \cos (\phi)-2 \phi^{2} \varphi \sin (\phi)+\phi \sin (\phi) \cos (\phi) \sin (\phi \varphi)-\sin (\phi) \cos (\phi)\left(\phi-\phi e^{\phi \varphi}\right) .
\end{array}
$$

As seen, all $S_{i}(\phi, \varphi), i=1,2,3$ are in $C^{1}(S, \mathbb{R})$. Consequently, $\gamma(\phi)$ is a line of curvature on $S(\phi, \varphi)$ with positive curvature and negative torsion .

By taking $R=[0,2 \pi] \times[-3,1]$, we visualize the curve $\gamma(\phi)=S(\phi, 0)$ in Fig.3.1, the surface $S(\phi, \varphi)$ in Fig. 3.2 and curve on surface in Fig. 3.3. 


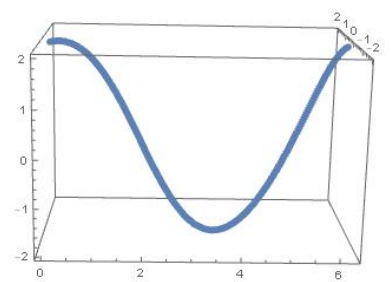

FIG. 3.1: Image of $\gamma(\phi)$

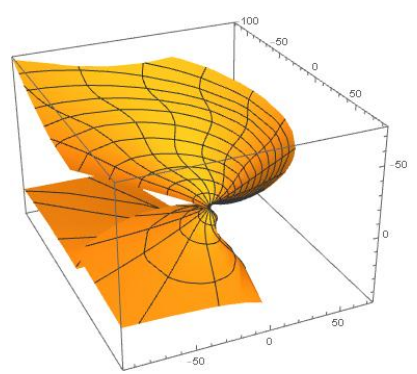

FIG. 3.2: Image of $S(\phi, \varphi)$

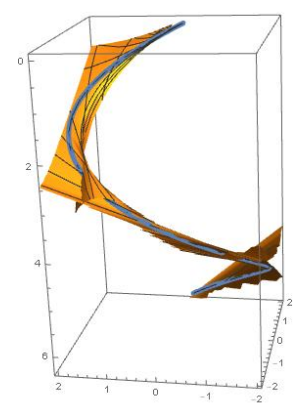

FIG. 3.3: $\gamma(\phi)$ on the $S(\phi, \varphi)$

Let take into consideration the case of the marching-scale functions

$$
\begin{aligned}
\lambda_{1}(\phi, \varphi) & =\rho_{1}(\phi) \Lambda_{1}(\varphi), \\
\lambda_{2}(\phi, \varphi) & =\rho_{2}(\phi) \Lambda_{2}(\varphi), \\
\lambda_{3}(\phi, \varphi) & =\rho_{3}(\phi) \Lambda_{3}(\varphi)
\end{aligned}
$$

with under conditions $\lambda_{1}\left(\phi, \varphi_{0}\right)=\lambda_{2}\left(\phi, \varphi_{0}\right)=\lambda_{3}\left(\phi, \varphi_{0}\right)=0$ and $\left(\phi, \varphi_{0}\right) \in R=$ $\left[I_{1}, I_{2}\right] \times\left[I_{3}, I_{4}\right]$, where $\rho_{1}(\phi), \Lambda_{1}(\varphi), \rho_{2}(\phi), \Lambda_{2}(\varphi), \rho_{3}(\phi)$ and $\Lambda_{3}(\varphi)$ are functions in $C^{1}(S, \mathbb{R})$. Then from Theorem 3.1 , we have following corollary:

Corollary 3.1. The curve $\gamma(\phi)=(\phi, \zeta(\phi), \psi(\phi))$ is a line of curvature on the surface defined in (2.6) if and only if

$$
\left\{\begin{array}{c}
\Lambda_{1}\left(\varphi_{0}\right)=\Lambda_{2}\left(\varphi_{0}\right)=\Lambda_{3}\left(\varphi_{0}\right)=0, \\
-\rho_{3}(\phi) \frac{d \Lambda_{3}}{d \varphi}\left(\varphi_{0}\right)=\mu(\phi) \cos \theta, \rho_{2}(\phi) \frac{d \Lambda_{2}}{d \varphi}\left(\varphi_{0}\right)=\mu(\phi) \sin \theta
\end{array}\right.
$$

where $\left(\phi, \varphi_{0}\right) \in R=\left[I_{1}, I_{2}\right] \times\left[I_{3}, I_{4}\right]$ and $\mu(\phi) \neq 0$.

Example 3.2. Let $\gamma(\phi)=(\phi, \cos (\phi), \sin (\phi))$ be an admissible curve in $\mathbb{G}_{3}$. Then, we get first two curvatures as $\kappa_{1}=1$ and $\kappa_{2}=1$. Also the Frenet frame is given by

$V_{1}(\phi)=(1,-\sin (\phi), \cos (\phi)), \quad V_{2}(\phi)=(0,-\cos (\phi),-\sin (\phi)), \quad V_{3}(\phi)=(0, \sin (\phi), \cos (\phi))$.

Thus, we get surface family $S(\phi, \varphi)$ given by (2.6) with common curve $\gamma(\phi)$. Suppose that the normal surface $\Psi(\phi, \varphi)$ of $S$ is developable in $\mathbb{G}_{3}$. Thus, from (2.8), we have $\theta=-\phi$.

If we choose $\rho_{1}(\phi)=\phi, \Lambda_{1}(\phi, \varphi)=\left(\varphi^{2}-1\right), \rho_{2}(\phi)=\rho_{3}(\phi)=1, \Lambda_{2}(\phi, \varphi)=\sin (\theta)(\varphi-$ 1), $\Lambda_{3}(\phi, \varphi)=\cos (\theta)(1-\varphi)$, and take $\mu(\phi)=1, \varphi_{0}=1$ so that equation (3.1) is satisfied, then a member of surface family in $\mathbb{G}_{3}$ is obtained by

$$
\begin{aligned}
S(\phi, \varphi)= & \left(\phi+\phi\left(\varphi^{2}-1\right), \cos (\phi)-\phi\left(\varphi^{2}-1\right) \sin (\phi),\right. \\
& \left.\sin (\phi)+\phi\left(\varphi^{2}-1\right) \cos (\phi)+\sin ^{2}(\phi)(\varphi-1)+\cos ^{2}(\phi)(1-\varphi)\right) .
\end{aligned}
$$

By taking $R=[0,2 \pi] \times[0,3]$, we visualize the curve $\gamma(\phi)=S(\phi, 0)$ in Fig.3.4, the surface $S(\phi, \varphi)$ in Fig. 3.5 and curve on surface in Fig. 3.6. 


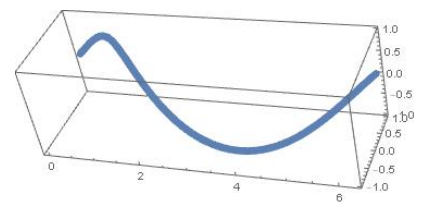

FIG. 3.4: Image of $\gamma(\phi)$

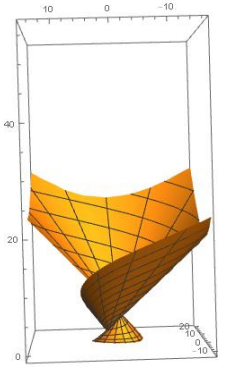

FIG. $\quad 3.5$ :

Image of $S(\phi, \varphi)$

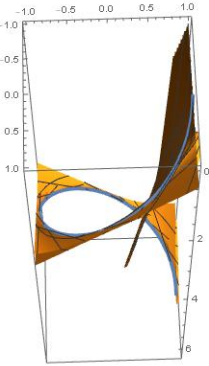

FiG. 3.6: $\gamma(\phi)$ on $S(\phi, \varphi)$

Suppose that the second curvature of $\gamma(\phi)$ vanish, i.e $\kappa_{2}=0$. Then, from (2.8), we have $\theta=\theta_{r}$ (constant). Thus, from (3.3), we obtain

$$
\frac{\mu(\phi)}{\rho_{3}(\phi)}=-c_{1}, \quad \frac{\mu(\phi)}{\rho_{2}(\phi)}=c_{2} .
$$

Considering conditions in (3.3), we get

$$
\frac{d \Lambda_{3}}{d \varphi}\left(\varphi_{0}\right)=c_{1} \cos \theta_{r} \text { and } \frac{d \Lambda_{2}}{d \varphi}\left(\varphi_{0}\right)=c_{2} \cos \theta_{r} .
$$

On the other hand, since $\mathfrak{n} \| \mathcal{N}$, if $\theta_{r}=(2 m+1) \frac{\pi}{2}$ for any integer $m$ then $V_{3} \| \mathcal{N}$. Thus, $\gamma(\phi)$ is an isoasymptotic curve on the surface. Also, if $\theta_{r}=m \pi$ then $V_{2} \| \mathcal{N}$ meaning $\gamma(\phi)$ is an isogeodesic curve on the surface. Consequently, we obtain the following result.

Corollary 3.2. Let the curve $\gamma(\phi)=(\phi, \zeta(\phi), \psi(\phi))$ be a line of curvature with torsion free on the surface is defined in (2.6). Then, we have

$$
\begin{aligned}
& \text { if } \theta_{r}=(2 m+1) \frac{\pi}{2} \text { for any integer } m \text {, then } \gamma(\phi) \text { is also isoasympotic, } \\
& \text { if } \theta_{r}=m \pi \text { for any integer } m \text {, then } \gamma(\phi) \text { is also isogeodesic. }
\end{aligned}
$$

Example 3.3. Let $\gamma(\phi)=(\phi, 1+\sin \phi, \sin \phi)$ be an admissible curve in $\mathbb{G}_{3}$. Then, we get first two curvatures as $\kappa_{1}=\sqrt{2} \sin \phi$ and $\kappa_{2}=0$. Also, the Frenet frame is given by

$$
V_{1}(\phi)=(1, \cos (\phi), \cos (\phi)), \quad V_{2}(\phi)=\left(0,-\frac{1}{\sqrt{2}},-\frac{1}{\sqrt{2}}\right), \quad V_{3}(\phi)=\left(0, \frac{1}{\sqrt{2}},-\frac{1}{\sqrt{2}}\right) .
$$

If we choose

$$
\rho_{1}(\phi)=\phi^{2}, \Lambda_{1}(\varphi)=\varphi, \quad \rho_{2}(\phi)=\phi, \Lambda_{2}(\varphi)=\varphi \sin \left(\theta_{r}\right), \quad \rho_{3}(\phi)=\phi, \Lambda_{3}(\phi)=-\varphi \cos \theta_{r}
$$


and take $\varphi_{0}=0, c_{1}=c_{2}=1$, then a member of surface family in $\mathbb{G}_{3}$ is obtained by

$$
\begin{aligned}
S(\phi, \varphi)= & \left(\phi+\phi^{2} \varphi, 1+\sin \phi-\frac{1}{\sqrt{2}} \phi \varphi \sin \left(\theta_{r}\right)-\frac{1}{\sqrt{2}} \phi \varphi \cos \left(\theta_{r}\right),\right. \\
& \left.\sin \phi-\frac{1}{\sqrt{2}} \phi \varphi \sin \theta_{r}+\frac{1}{\sqrt{2}} \phi \varphi \cos \theta_{r}\right) .
\end{aligned}
$$

By taking $R=[0,2 \pi] \times[0,0.2]$, we visualize the curve $\gamma(\phi)=S(\phi, 0)$ in Fig.3.7 and

1. the surface $S(\phi, \varphi)$ in Fig. 3.8 for $\theta=\frac{\pi}{6}$;

2. curve on surface in Fig. 3.9 for $\theta=\frac{\pi}{6}$, in Fig. 3.10 for $\theta=\frac{\pi}{2}$; in Fig. 3.11 for $\theta=0$.

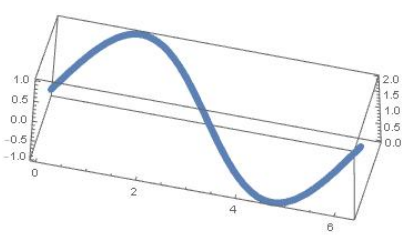

FIG. 3.7: Image of $\gamma(\phi)$

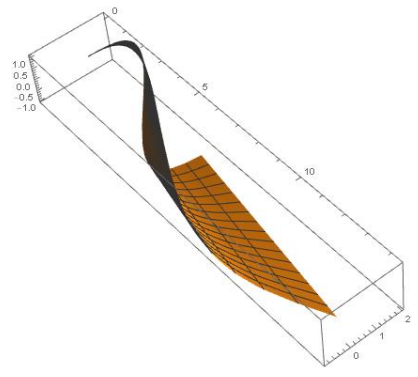

FIG. 3.8: Image of $S(\phi, \varphi)$

FIG. 3.9: $\gamma(\phi)$ on $S(\phi, \varphi)$

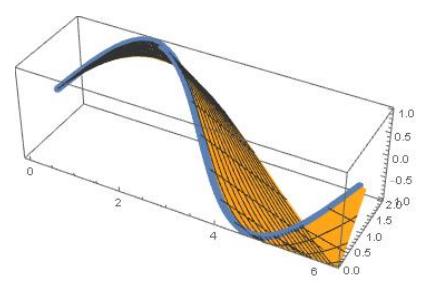

FIG. 3.10: $\gamma(\phi)$ on $S(\phi, \varphi)$
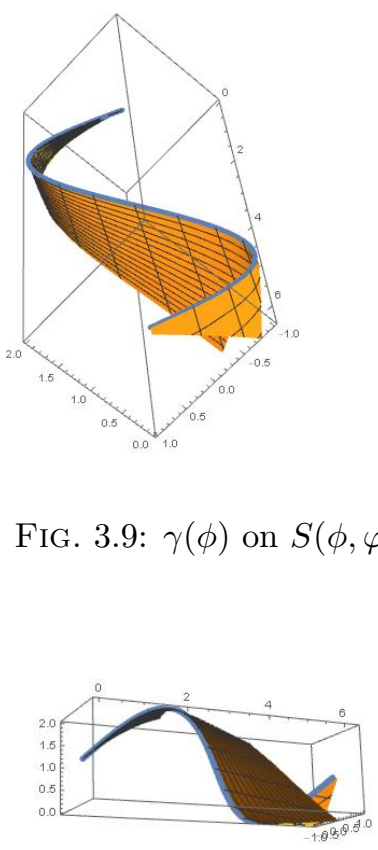

FIG. 3.11: $\gamma(\phi)$ on $S(\phi, \varphi)$

\section{Acknowledgements}

The authors would like to thank Dr. M. Evren AYDIN for his helpful suggestions and valuable comments which helped to improve the manuscript. 


\section{R E F E R E N C E S}

1. G.S. AtAlay AND E. KASAP: Family of surface with a common null geodesic. International Journal of Physical Sciences 4(8) (2009), 428-433.

2. G.Ş. AtAlay AND E. KASAP: Surfaces family with common null asymptotic Applied Mathematics and Computation 260 (2015), 135-139.

3. M.E. Aydin, M.A. Külahçı AND A.O. ÖĞRenmiş: Constant Curvature Translation Surfaces in Galilean 3-Space International Electronic Journal of Geometry 12(1) (2019), 9-19.

4. W. Che, J-C. Paul And X. Zhang: Lines of curvature and umbilical points for implicit surfaces Computer Aided Geometric Design 24(7) (2007), 395-409.

5. M. DEDE :Tubular surfaces in Galilean space Mathematical Communications 18(1) (2013), 209-217.

6. B. Divjak, Z. Milin-Sipus: Special curves on ruled surfaces in Galilean and pseudo-Galilean space Acta Math. Hungar. 98 (2003), 203-215.

7. E. Ergün, E. BAyRAm, AND E. KASAP : Surface pencil with a common line of curvature in Minkowski 3-space Acta Mathematica Sinica, English Series 30(12) (2014), 2103-2118.

8. E. ERGüN, E. BAYRAM, AND E. KASAP : Surface family with a common natural line of curvature lift Journal of Science and Arts 15(4) (2015), 321.

9. E. KASAP AND F.T. AKYILDIZ: Surfaces with common geodesic in Minkowski 3-space Applied mathematics and computation 177(1) (2006), 260-270.

10. E. KASAP, F.T. AKYILDIZ AND K. ORBAY: A generalization of surfaces family with common spatial geodesic Applied Mathematics and Computation 201(1-2) (2008), 781-789.

11. C-Y. LI, R-H. WANG AND C-G ZHU: Parametric representation of a surface pencil with a common line of curvature Computer-Aided Design 43(9) (2011), 1110-1117.

12. C-Y. Li, R-H. WANG, AND C-G ZHU: A generalization of surface family with common line of curvature Applied Mathematics and Computation 219(17) (2013), 9500-9507.

13. C-Y. LI, R-H. WANG, AND C-G ZHU: Designing approximation minimal parametric surfaces with geodesics Applied mathematical modelling 37(9) (2013), 6415-6424.

14. Z.E. MusielaK AND J. L. FRY : Physical theories in Galilean space-time and the origin of Schrödinger-like equations Annals of Physics, 324-2 (2009), 296-308.

15. A.O. Ögrenmis, M. Ergut and M. Bektas :On the helices in the Galilean space $\mathbb{G}_{3}$ Iran.J. Sci. Technol. Trans. A Sci. 31(2) (2007), 177-181.

16. A.O. ÖĞREnmiş, H. Öztekin And M. Ergüt: Bertrand curves in Galilean space and their characterizations Kragujevac Journal of Mathematics 32(32) (2009), 139-147.

17. H. Öztekin : Special Bertrand curves in $4 D$ Galilean space Mathematical Problems in Engineering Hindawi Publishing Corporation 2014 (2014), 1-7.

18. O. Röschel, :Die geometrie des Galileischen raumes Forschungszentrum Graz Mathematisch-Statistische Sektion, Graz. 1(2) (1985). 
19. D.J. StRuiK: Lectures on classical differential geometry Courier Corporation, 1961.

20. I.M. YAGLOM: A simple non-Euclidean geometry and its physical basis: An elementary account of Galilean geometry and the Galilean principle of relativit Springer Science Business Media, 2012.

21. D.W. Yoon, J.W. LeE AND C.W. LEE : Osculating curves in the Galilean 4-Space International Journal of Pure and Applied Mathematics 100(4) (2015) 497-506.

22. D.W. YOON AND Z.K. YÜZBASI : An approach for hypersurface family with common geodesic curve in the $4 D$ Galilean space $\mathbb{G}_{4}$, The Pure and Applied Mathematics 25(4) (2018) 229-241.

23. Z.K. YÜZBAŞI AND M. BEKTAŞ : On the construction of a surface family with common geodesic in Galilean space $\mathbb{G}_{3}$ Open Physics 14(1) (2016) 360-363.

24. Z.K. YÜZBASI : On a family of surfaces with common asymptotic curve in the Galilean space $\mathbb{G}_{3}$, J. Nonlinear Sci. Appl. 9 (2016) 518-523.

25. G-J. WANG, K. TANG AND C-L. TAI : Parametric representation of a surface pencil with a common spatial geodesic Computer-Aided Design 36(5) (2004) 447459 .

\author{
Mustafa Altin \\ Technical Sciences Vocational School \\ Bingol University \\ 12000 Bingöl, Turkey \\ maltin@bingol.edu.tr \\ İnan Ünal \\ Department of Computer Engineering \\ Munzur University \\ 62000 Tunceli, Turkey \\ inanunal@munzur.edu.tr
}

